\title{
A finite element method with discontinuous rotations for the Mindlin-Reissner plate model
}

\author{
Peter Hansbo, ${ }^{\text {a }}$ David Heintz, ${ }^{\text {a }}$ Mats G. Larson ${ }^{\text {b }}$ \\ ${ }^{a}$ Department of Mathematical Sciences, Chalmers University of Technology and \\ University of Gothenburg, SE-41296 Göteborg, Sweden \\ ${ }^{\mathrm{b}}$ Department of Mathematics and Mathematical Statistics, Umeå University, SE-901 87 \\ Umeå, Sweden
}

\begin{abstract}
We present a continuous-discontinuous finite element method for the Mindlin-Reissner plate model based on continuous polynomials of degree $k \geq 2$ for the transverse displacements and discontinuous polynomials of degree $k-1$ for the rotations. We prove a priori convergence estimates, uniformly in the thickness of the plate, and thus show that locking is avoided. We also derive a posteriori error estimates based on duality, together with corresponding adaptive procedures for controlling linear functionals of the error. Finally, we present some numerical results.
\end{abstract}

Key words: Nitsche's method, discontinuous Galerkin, plate model, error estimates.

\section{Introduction}

Plates are very common simplified models for thin structures in engineering practice. The most basic plate models are the Kirchhoff model, which is a fourth order partial differential equation, and the Mindlin-Reissner (MR) model, which is a system of second order partial differential equations. The Kirchhoff model can be seen as the limit of the MR model as the thickness of the plate tends to zero. Finite element approximations of plate models would seem to be easier to handle with the MR model, since then only $C^{0}$ continuity is required, as opposed to the $C^{1}$ continuous elements needed for the Kirchhoff model. However, in order for a finite element method to work asymptotically as $t \rightarrow 0$ in the MR model, typically rather complicated approximations must be used.

In this paper, we will consider a family of simple continuous-discontinuous Galerkin finite element methods for the MR model, first proposed in [10], based on discon- 
tinuous piecewise polynomials for the discretization of the rotations and continuous piecewise polynomials of one degree higher for the transverse displacements.

When the thickness of the plate tends to zero we obtain the Kirchhoff plate and our scheme simplifies to the method proposed in [9]. In this context we also mention the discontinuous Galerkin methods for the Kirchhoff plate developed by Hansbo and Larson [12] and for the Mindlin-Reissner model by Bösing, Madureira, and Mozolevski [4].

\section{The continuous problem}

The Mindlin-Reissner plate model is described by the following partial differential equations:

$$
\begin{aligned}
-\nabla \cdot \boldsymbol{\sigma}(\boldsymbol{\theta})-\kappa t^{-2}(\nabla u-\boldsymbol{\theta}) & =0, & & \text { in } \Omega \subset \mathbb{R}^{2}, \\
-\kappa t^{-2} \nabla \cdot(\nabla u-\boldsymbol{\theta}) & =g, & & \text { in } \Omega,
\end{aligned}
$$

where $u$ is the transverse displacement, $\boldsymbol{\theta}$ is the rotation of the median surface, $t$ is the thickness, assumed constant, $t^{3} g$ is the transverse surface load, and

$$
\boldsymbol{\sigma}(\boldsymbol{\theta}):=2 \mu \varepsilon(\boldsymbol{\theta})+\lambda \nabla \cdot \boldsymbol{\theta} \mathbf{1}
$$

is the moment tensor. Here, 1 is the identity tensor and $\varepsilon$ is the strain operator with components

$$
\varepsilon_{i j}(\boldsymbol{\theta})=\frac{1}{2}\left(\frac{\partial \theta_{i}}{\partial x_{j}}+\frac{\partial \theta_{j}}{\partial x_{i}}\right) .
$$

The material constants are given by the relations

$$
\kappa:=\frac{E k}{2(1+\nu)}, \mu:=\frac{E}{24(1+\nu)}, \lambda:=\frac{\nu E}{12\left(1-\nu^{2}\right)},
$$

where $E$ and $\nu$ are the Young's modulus and Poisson's ratio, respectively, and $k$ is a shear correction factor usually set to $k=5 / 6$. For simplicity, we shall assume that the domain $\Omega$ is a convex polygon and consider the case of clamped boundary conditions: $\boldsymbol{\theta}=\mathbf{0}$ and $u=0$ on $\partial \Omega$.

The differential equations describing the MR plate model can be derived from minimization of the sum of the bending energy, the shear energy, and the potential of the surface load,

$$
\mathfrak{F}(u, \boldsymbol{\theta}):=\frac{1}{2} a(\boldsymbol{\theta}, \boldsymbol{\theta})+\frac{1}{2} b(u, \boldsymbol{\theta} ; u, \boldsymbol{\theta})-(g, u)_{\Omega} .
$$

where $(\cdot, \cdot)_{\Omega}$ denotes the $L_{2}$ scalar product over the indicated domain, the bending energy $a(\cdot, \cdot)$ is defined by

$$
a(\boldsymbol{\theta}, \boldsymbol{\vartheta}):=(\boldsymbol{\sigma}(\boldsymbol{\theta}), \boldsymbol{\varepsilon}(\boldsymbol{\vartheta}))_{\Omega},
$$


and the shear energy $b(\cdot, \cdot ; \cdot, \cdot)$ by

$$
b(u, \boldsymbol{\theta} ; v, \boldsymbol{\vartheta}):=\frac{\kappa}{t^{2}}(\nabla u-\boldsymbol{\theta}, \nabla v-\boldsymbol{\vartheta})_{\Omega} .
$$

The difficulty with this model, from a numerical point of view, is the matching of the approximating spaces for $\boldsymbol{\theta}$ and $u$. As $t \rightarrow 0$, the difference $\nabla u-\boldsymbol{\theta}$ must tend to zero; if this is not allowed by the approximating spaces the result is a deterioration of the numerical solution known as shear locking (the shear energy increases without bound). The situation is particularly difficult if we wish to use low order approximations. One useful approach has been to use projections in the shear energy term and consider modified energy functionals of the type

$$
\mathfrak{F}_{h}(u, \boldsymbol{\theta}):=\frac{1}{2} a(\boldsymbol{\theta}, \boldsymbol{\theta})+\frac{1}{2} b\left(u, \boldsymbol{R}_{h} \boldsymbol{\theta} ; u, \boldsymbol{R}_{h} \boldsymbol{\theta}\right)-(g, u)_{\Omega},
$$

where $\boldsymbol{R}_{h}$ is some interpolation or projection operator. This idea underpins, e.g., the MITC element family of Bathe and co-workers, first introduced in [2], and has been used extensively in the mathematical literature to prove convergence, see, e.g., $[1,6,8,14]$. It should be noted that if the approximation corresponding to $\boldsymbol{R}_{h} \boldsymbol{\theta}$ were to be used also for the bending energy, the element would be non-conforming, and potentially unstable. This means that we in effect have to construct and match three different finite element spaces, and this is indeed how the approach was originally conceived: as a mixed method with an auxiliary set of unknowns (the shear stresses), cf. [2].

\section{The finite element method}

For simplicity, we shall consider the case of clamped boundary conditions. The transverse displacement and rotation vector are solutions to the following variational problem: find $\boldsymbol{\theta} \in\left[H_{0}^{1}(\Omega)\right]^{2}$ and $u \in H_{0}^{1}(\Omega)$ such that

$$
a(\boldsymbol{\theta}, \boldsymbol{\vartheta})+b(u, \boldsymbol{\theta} ; v, \boldsymbol{\vartheta})=(g, v)_{\Omega}
$$

for all $(v, \boldsymbol{\vartheta}) \in H_{0}^{1}(\Omega) \times\left[H_{0}^{1}(\Omega)\right]^{2}$. However, as is common in discontinuous Galerkin methods, we need to assume a higher regularity of the solution in order for all terms in our scheme to make sense with the exact solution inserted. In particular, we will need $\boldsymbol{n} \cdot \boldsymbol{\sigma}(\boldsymbol{\theta})$ to be in $L_{2}$ on each edge (with normal $\boldsymbol{n}$ ) of the mesh. Since the error estimates in fact require even more regularity, we will in the following simply assume that $\boldsymbol{\theta} \in\left[H_{0}^{1}(\Omega)\right]^{2} \cap\left[H^{2}(\Omega)\right]^{2}$ and $u \in H_{0}^{1}(\Omega) \cap H^{2}(\Omega)$.

To define the method, consider a subdivision $\mathfrak{T}=\{T\}$ of $\Omega$ into a geometrically conforming, quasiuniform, finite element mesh of affine triangles. Denote by $h_{T}$ the diameter of element $T$ and by $h=\max _{T \in \mathfrak{T}} h_{T}$ the global mesh size parameter. We shall use continuous, piecewise polynomial, approximations of the transverse 
displacement,

$$
V_{h}=\left\{v \in H^{1}(\Omega):\left.v\right|_{T} \in P^{k}(T) \text { for all } T \in \mathfrak{T}, v=0 \text { on } \partial \Omega\right\},
$$

and discontinuous polynomials for the rotations:

$$
\boldsymbol{\Theta}_{h}:=\left\{\boldsymbol{\vartheta} \in\left[L^{2}(\Omega)\right]^{2}:\left.\boldsymbol{\vartheta}\right|_{T} \in\left[P^{k-1}(T)\right]^{2} \text { for all } T \in \mathfrak{T}\right\}
$$

where $k \geq 2$.

We note that the approximating spaces are compatible in the sense that

$$
\nabla v \in \boldsymbol{\Theta}_{h}, \quad \forall v \in V_{h} .
$$

In the limit $t \rightarrow 0$, functions in $\Theta_{h}$ are then allowed to belong to $\nabla V_{h}$ which alleviates locking.

Remark 1 The case of quadrilateral elements is not equally easy to handle in the general isoparametric case: the gradients of functions on isoparametrically mapped elements are not polynomials, which makes the relation (5) more delicate to fulfill.

To define our method we introduce the set of edges in the mesh, $\mathfrak{E}=\{E\}$, and we split $\mathfrak{E}$ into two disjoint subsets

$$
\mathfrak{E}=\mathfrak{E}_{I} \cup \mathfrak{E}_{B},
$$

where $\mathfrak{E}_{I}$ is the set of edges in the interior of $\Omega$ and $\mathfrak{E}_{B}$ is the set of edges on the boundary. Further, with each edge we associate a fixed unit normal $\boldsymbol{n}$ such that for edges on the boundary $\boldsymbol{n}$ is the exterior unit normal. We denote the jump of a function $v \in V_{h}$ (and analogously for $\boldsymbol{v} \in \boldsymbol{\Theta}_{h}$ ) at an edge $E$ by $[v]=v^{+}-v^{-}$for $E \in \mathfrak{E}_{I}$ and $[v]=v^{+}$for $E \in \mathfrak{E}_{B}$, and the average $\langle v\rangle=\left(v^{+}+v^{-}\right) / 2$ for $E \in \mathfrak{E}_{I}$ and $\langle v\rangle=v^{+}$for $E \in \mathfrak{E}_{B}$, where $v^{ \pm}=\lim _{\epsilon \downarrow 0} v(\boldsymbol{x} \mp \epsilon \boldsymbol{n})$ with $\boldsymbol{x} \in E$.

Our method can now be formulated as follows: find $\boldsymbol{\theta}^{h} \in \Theta_{h}$ and $u^{h} \in V_{h}$ such that

$$
a_{h}\left(\boldsymbol{\theta}^{h}, \boldsymbol{\vartheta}\right)+b\left(u^{h}, \boldsymbol{\theta}^{h} ; v, \boldsymbol{\vartheta}\right)=(g, v)_{\Omega}
$$

for all $(v, \boldsymbol{\vartheta}) \in V_{h} \times \boldsymbol{\Theta}_{h}$. In (6), the bilinear form $a_{h}(\cdot, \cdot)$ is defined by

$$
\begin{aligned}
a_{h}\left(\boldsymbol{\theta}^{h}, \boldsymbol{\vartheta}\right)= & \sum_{T \in \mathfrak{T}}\left(\boldsymbol{\sigma}\left(\boldsymbol{\theta}^{h}\right), \boldsymbol{\varepsilon}(\boldsymbol{\vartheta})\right)_{T}-\sum_{E \in \mathfrak{E}}\left(\left\langle\boldsymbol{n} \cdot \boldsymbol{\sigma}\left(\boldsymbol{\theta}^{h}\right)\right\rangle,[\boldsymbol{\vartheta}]\right)_{E} \\
& -\sum_{E \in \mathfrak{E}}\left(\langle\boldsymbol{n} \cdot \boldsymbol{\sigma}(\boldsymbol{\vartheta})\rangle,\left[\boldsymbol{\theta}^{h}\right]\right)_{E} \\
& +(2 \mu+2 \lambda) \gamma \sum_{E \in \mathfrak{E}}\left(h_{E}^{-1}\left[\boldsymbol{\theta}^{h}\right],[\boldsymbol{\vartheta}]\right)_{E} .
\end{aligned}
$$


Here $\gamma$ is a positive constant and $h_{E}$ is defined by

$$
h_{E}=\left(\left|T^{+}\right|+\left|T^{-}\right|\right) /(2|E|) \text { for } E=\partial T^{+} \cap \partial T^{-},
$$

with $|T|$ the area of $T$, on each edge.

Using Green's formula, we readily establish the following Lemma.

Lemma 2 The method (6) is consistent in the sense that

$$
a_{h}\left(\boldsymbol{\theta}-\boldsymbol{\theta}^{h}, \boldsymbol{\vartheta}\right)+b\left(u-u^{h}, \boldsymbol{\theta}-\boldsymbol{\theta}^{h} ; v, \boldsymbol{\vartheta}\right)=0
$$

for all $\boldsymbol{\vartheta} \in \boldsymbol{\Theta}_{h}$ and $v \in V_{h}$.

\section{Stability estimates}

For our analysis, we introduce the following edge norm

$$
\|\boldsymbol{\vartheta}\|_{\mathfrak{E}}^{2}=\sum_{E \in \mathfrak{E}}\|\boldsymbol{\vartheta}\|_{L^{2}(E)}^{2} .
$$

and mesh dependent energy-like norm

$$
\begin{aligned}
\|\| \boldsymbol{\vartheta}\|\|^{2}= & \sum_{T \in \mathfrak{T}}(\boldsymbol{\sigma}(\boldsymbol{\vartheta}), \boldsymbol{\varepsilon}(\boldsymbol{\vartheta}))_{T}+\frac{1}{2 \mu+2 \lambda}\left\|h_{E}^{1 / 2}\langle\boldsymbol{n} \cdot \boldsymbol{\sigma}(\boldsymbol{\vartheta})\rangle\right\|_{\mathfrak{E}}^{2} \\
& +(2 \mu+2 \lambda)\left\|h_{E}^{-1 / 2}[\boldsymbol{\vartheta}]\right\|_{\mathfrak{E}}^{2},
\end{aligned}
$$

The mesh dependent norm $\||\cdot|||$ can be used to bound the broken $H^{1}(\Omega)$ norm on $\Theta_{h}$, which is the statement of the following Lemma.

Lemma 3 There is a constant $c$, independent of $h, \mu$, and $\lambda$ such that

$$
\sum_{T \in \mathfrak{T}}\|\boldsymbol{\vartheta}\|_{H^{1}(T)}^{2} \leq c\|\| \boldsymbol{\vartheta} \|^{2} \quad \text { for all } \boldsymbol{\vartheta} \in \boldsymbol{\Theta}_{h}
$$

PROOF. This is a discrete Korn-type inequality that results from the control of the rigid body rotations given by the jump terms. A complete proof can be found in [5].

In order to show that the method (6) is stable, we shall first show that $a_{h}(\cdot, \cdot)$ is coercive with respect to the norm $\||\cdot|\|$, given that $\gamma$ is sufficiently large.

Lemma 4 If $\gamma>c_{0}$, with $c_{0}$ sufficiently large, then the following estimate holds

$$
c \mid\|\boldsymbol{\vartheta}\| \|^{2} \leq a_{h}(\boldsymbol{\vartheta}, \boldsymbol{\vartheta}),
$$


for all $\boldsymbol{v} \in \Theta_{h}$.

PROOF. We first note that the following inverse estimate holds

$$
\left\|h^{1 / 2}\langle\boldsymbol{n} \cdot \boldsymbol{\sigma}(\boldsymbol{\vartheta})\rangle\right\|_{\mathfrak{E}}^{2} \leq c_{I} \sum_{T \in \mathfrak{T}}\|\boldsymbol{\sigma}(\boldsymbol{\vartheta})\|_{L_{2}(T)}^{2} .
$$

This inequality is proved by scaling and finite dimensionality (see, e.g. [15]). Next we note that

$$
\frac{1}{2 \mu+2 \lambda}\|\boldsymbol{\sigma}(\boldsymbol{\vartheta})\|_{L_{2}(T)}^{2} \leq(\boldsymbol{\sigma}(\boldsymbol{\vartheta}), \boldsymbol{\varepsilon}(\boldsymbol{\vartheta}))_{T},
$$

cf. Hansbo and Larson [11], and thus we conclude that

$$
\frac{1}{2 \mu+2 \lambda}\left\|h^{1 / 2}\langle\boldsymbol{n} \cdot \boldsymbol{\sigma}(\boldsymbol{\vartheta})\rangle\right\|_{\mathfrak{E}}^{2} \leq c_{I} \sum_{T \in \mathfrak{T}}(\boldsymbol{\sigma}(\boldsymbol{\vartheta}), \boldsymbol{\varepsilon}(\boldsymbol{\vartheta}))_{T} .
$$

Next, we have, for each $E \in \mathfrak{E}$, that

$$
\begin{aligned}
2(\langle\boldsymbol{n} \cdot \boldsymbol{\sigma}(\boldsymbol{\vartheta})\rangle,[\boldsymbol{\vartheta}])_{E} \leq & \delta(2 \mu+2 \lambda)^{-1}\left\|h^{1 / 2}\langle\boldsymbol{n} \cdot \boldsymbol{\sigma}(\boldsymbol{\vartheta})\rangle\right\|_{L_{2}(E)}^{2} \\
& +\delta^{-1}(2 \mu+2 \lambda)\left\|h^{-1 / 2}[\boldsymbol{\vartheta}]\right\|_{L_{2}(E)}^{2}
\end{aligned}
$$

where we used the Cauchy-Schwarz inequality followed by the arithmetic-geometric mean inequality. Using these estimates and choosing, e.g., $\delta=\left(4 c_{I}\right)^{-1}$, we obtain

$$
\begin{aligned}
a_{h}(\boldsymbol{\vartheta}, \boldsymbol{\vartheta}) \geq & \frac{1}{2} \sum_{T \in \mathfrak{T}}(\boldsymbol{\sigma}(\boldsymbol{\vartheta}), \boldsymbol{\varepsilon}(\boldsymbol{\vartheta}))_{T} \\
& +(2 \mu+2 \lambda)\left(\gamma-2 c_{I}\right)\left\|h^{-1 / 2}[\boldsymbol{\vartheta}]\right\|_{\mathfrak{E}}^{2} \\
& +\frac{1}{4 c_{I}}(2 \mu+2 \lambda)^{-1}\left\|h^{1 / 2}\langle\boldsymbol{n} \cdot \boldsymbol{\sigma}(\boldsymbol{\vartheta})\rangle\right\|_{\mathfrak{E}}^{2} \\
\geq & c\|\boldsymbol{\vartheta}\| \|^{2},
\end{aligned}
$$

whence we must choose $\gamma \geq c_{0}>4 c_{I}$.

We have thus shown the following stability property of the method.

Proposition 5 Choosing $\gamma \geq c_{0}>4 c_{I}$, the following coercivity condition holds:

$$
a_{h}(\boldsymbol{\vartheta}, \boldsymbol{\vartheta})+\frac{\kappa}{t^{2}} \int_{\Omega}|\nabla v-\boldsymbol{\vartheta}|^{2} d \Omega \geq C\left(\left\|\boldsymbol{\vartheta}\left|\left\|\mid+\kappa^{1 / 2} t^{-1}\right\| \nabla v-\boldsymbol{\vartheta} \|_{L_{2}(\Omega)}\right)^{2},\right.\right.
$$

for all $(\boldsymbol{\vartheta}, v) \in \Theta_{h} \times V_{h}$.

We finally remark that the constant $c_{I}$ in the inverse estimate (13) is computable and thus the lower bound $c_{0}$ on $\gamma$ is available, see [13] for details. 


\section{A priori error estimates}

In this section, we will derive a priori error estimates for CDG methods in the case $k=2$, and show that they hold uniformly in $t$. For higher order methods, edge effects will typically preclude global estimates because of the lack of regularity of the exact solution.

\subsection{An estimate in energy norm}

For convenience, we introduce the scaled shear stress $\zeta$ and its discrete counterpart $\zeta^{h}$, defined by

$$
\boldsymbol{\zeta}:=\kappa^{1 / 2}(\nabla u-\boldsymbol{\theta}) / t^{2} \quad \text { and } \quad \boldsymbol{\zeta}^{h}:=\kappa^{1 / 2}\left(\nabla u^{h}-\boldsymbol{\theta}^{h}\right) / t^{2} .
$$

We also split the Mindlin-Reissner displacement $u$ into the corresponding Kirchhoff solution $u_{0}$ corresponding to the limit case $t \rightarrow 0$, and a remainder $u_{r}$, so that $u=u_{0}+u_{r}$. We then have the following stability estimate.

Lemma 6 Assume that $\Omega$ is convex and $g \in L_{2}(\Omega)$. Then

$$
\left\|u_{0}\right\|_{H^{3}(\Omega)}+\frac{1}{t}\left\|u_{r}\right\|_{H^{2}(\Omega)}+\|\boldsymbol{\theta}\|_{H^{2}(\Omega)}+t\|\boldsymbol{\zeta}\|_{H^{1}(\Omega)} \leq C\left(\|g\|_{H^{-1}(\Omega)}+t\|g\|_{L_{2}(\Omega)}\right) .
$$

For a proof, see $[7,1]$.

For the purpose of analysis, we introduce the nodal interpolation operators $\boldsymbol{\pi}_{1}$ : $\left[H^{2}(\Omega)\right]^{2} \rightarrow \boldsymbol{W}_{h}$, where

$$
\boldsymbol{W}_{h}:=\left\{\boldsymbol{v} \in\left[H^{1}(\Omega) \cap C^{0}(\Omega)\right]^{2}:\left.\boldsymbol{v}\right|_{T} \in\left[P^{1}(T)\right]^{2} \text { for all } T \in \mathfrak{T}\right\},
$$

and $\pi_{2}: H^{2}(\Omega) \rightarrow V_{h}$. We also define the operators $\boldsymbol{P}_{u}:\left[H^{2}(\Omega)\right]^{2} \rightarrow \boldsymbol{\Theta}_{h}$ and $\boldsymbol{Q}_{u}:\left[H^{2}(\Omega)\right]^{2} \rightarrow \boldsymbol{\Theta}_{h}$ defined by

$$
\boldsymbol{P}_{u} \boldsymbol{\theta}:=\nabla \pi_{2} u_{0}-\boldsymbol{\pi}_{1} \nabla u_{0}+\boldsymbol{\pi}_{1} \boldsymbol{\theta}
$$

and

$$
\left.\boldsymbol{Q}_{u} \boldsymbol{\zeta}:=\kappa^{1 / 2}\left(\nabla \pi_{2} u_{r}-\boldsymbol{\pi}_{1} \nabla u_{r}\right)\right) / t^{2}+\boldsymbol{\pi}_{1} \boldsymbol{\zeta}
$$

Noting that

$$
\frac{t^{2}}{\kappa^{1 / 2}} \boldsymbol{Q}_{u} \boldsymbol{\zeta}=\nabla \pi_{2} u_{r}-\boldsymbol{\pi}_{1} \nabla u_{r}+\boldsymbol{\pi}_{1} \nabla\left(u_{r}+u_{0}\right)+\boldsymbol{\pi}_{1} \boldsymbol{\theta}=\nabla \pi_{2} u-\boldsymbol{P}_{u} \boldsymbol{\theta},
$$

and using Lemma 2, we then find

$$
a_{h}\left(\boldsymbol{\theta}-\boldsymbol{\theta}^{h}, \boldsymbol{P}_{u} \boldsymbol{\theta}\right)+t^{2}\left(\boldsymbol{\zeta}-\boldsymbol{\zeta}^{h}, \boldsymbol{Q}_{u} \boldsymbol{\zeta}\right)_{\Omega}=0 .
$$


We will need the following approximation properties of our finite element subspaces.

Lemma 7 We have the following interpolation estimate:

$$
\begin{gathered}
\|\| \boldsymbol{\theta}-\boldsymbol{P}_{u} \boldsymbol{\theta}\|\mid+t\| \boldsymbol{\zeta}-\boldsymbol{Q}_{u} \boldsymbol{\zeta} \|_{L_{2}(\Omega)} \leq C h\left(\|\boldsymbol{\theta}\|_{H^{2}(\Omega)}+\left\|u_{0}\right\|_{H^{3}(\Omega)}+t^{-1}\left\|u_{r}\right\|_{H^{2}(\Omega)}\right. \\
\left.+t\|\boldsymbol{\zeta}\|_{H^{1}(\Omega)}\right) .
\end{gathered}
$$

PROOF. We first recall the trace inequality (cf. [15])

$$
h_{T}^{-1}\|\boldsymbol{\vartheta}\|_{L_{2}(\partial T)}^{2} \leq C\left(h_{T}^{-2}\|\boldsymbol{\vartheta}\|_{L_{2}(T)}^{2}+\|\boldsymbol{\vartheta}\|_{H^{1}(T)}^{2}\right),
$$

for all $\vartheta \in\left[H^{2}(T)\right]^{2}$. For the edge norm we have that

$$
h_{E}^{-1}\left\|\left[\boldsymbol{\theta}-\boldsymbol{P}_{u} \boldsymbol{\theta}\right]\right\|_{L_{2}(E)}^{2} \leq C h_{E}^{-1}\left(\left\|\boldsymbol{\theta}-\boldsymbol{P}_{u} \boldsymbol{\theta}\right\|_{L_{2}\left(\partial T_{1}\right)}^{2}+\left\|\boldsymbol{\theta}-\boldsymbol{P}_{u} \boldsymbol{\theta}\right\|_{L_{2}\left(\partial T_{2}\right)}^{2}\right)
$$

for $E$ shared by adjacent elements $T_{1}$ and $T_{2}$, and since, by quasiuniformity, $h_{T_{i}} \leq$ $h_{E} / C, i=1,2$, we find, using (18),

$$
\begin{aligned}
h_{E}^{-1}\left\|\boldsymbol{\theta}-\boldsymbol{P}_{u} \boldsymbol{\theta}\right\|_{L_{2}\left(\partial T_{i}\right)}^{2} & \leq C h_{T_{i}}^{-1}\left\|\boldsymbol{\theta}-\boldsymbol{P}_{u} \boldsymbol{\theta}\right\|_{L_{2}\left(\partial T_{i}\right)}^{2} \\
& \leq C\left(h_{T_{i}}^{-2}\left\|\boldsymbol{\theta}-\boldsymbol{P}_{u} \boldsymbol{\theta}\right\|_{L_{2}\left(T_{i}\right)}^{2}+\left\|\boldsymbol{\theta}-\boldsymbol{P}_{u} \boldsymbol{\theta}\right\|_{H^{1}\left(T_{i}\right)}^{2}\right) .
\end{aligned}
$$

Using the definition of $\boldsymbol{P}_{u}$ and applying the triangle inequality, we find

$$
\left\|\boldsymbol{\theta}-\boldsymbol{P}_{u} \boldsymbol{\theta}\right\| \leq\left\|\boldsymbol{\theta}-\boldsymbol{\pi}_{1} \boldsymbol{\theta}\right\|+\left\|\nabla u_{0}-\nabla \pi_{2} u_{0}\right\|+\left\|\nabla u_{0}-\boldsymbol{\pi}_{1} \nabla u_{0}\right\|,
$$

so that, by standard interpolation theory,

$$
h_{E}^{-1}\left\|\boldsymbol{\theta}-\boldsymbol{P}_{u} \boldsymbol{\theta}\right\|_{L_{2}\left(\partial T_{i}\right)}^{2} \leq C h_{T}^{2}\left(\|\boldsymbol{\theta}\|_{H^{2}\left(T_{i}\right)}^{2}+\left\|u_{0}\right\|_{H^{3}\left(T_{i}\right)}^{2}\right) .
$$

Similarly,

$$
\begin{aligned}
h_{E}\left\|\boldsymbol{\sigma}\left(\boldsymbol{\theta}-\boldsymbol{P}_{u} \boldsymbol{\theta}\right)\right\|_{L_{2}\left(\partial T_{i}\right)}^{2} & \leq C\left(\left\|\boldsymbol{\theta}-\boldsymbol{P}_{u} \boldsymbol{\theta}\right\|_{H^{1}\left(T_{i}\right)}^{2}+h_{T_{i}}^{2}\left\|\boldsymbol{\theta}-\boldsymbol{P}_{u} \boldsymbol{\theta}\right\|_{H^{2}\left(T_{i}\right)}^{2}\right) \\
& \leq C h_{T_{i}}^{2}\left(\|\boldsymbol{\theta}\|_{H^{2}\left(T_{i}\right)}^{2}+\left\|u_{0}\right\|_{H^{3}\left(T_{i}\right)}^{2}\right)
\end{aligned}
$$

and

$$
\left(\boldsymbol{\sigma}\left(\boldsymbol{\theta}-\boldsymbol{P}_{u} \boldsymbol{\theta}\right), \boldsymbol{\varepsilon}\left(\boldsymbol{\theta}-\boldsymbol{P}_{u} \boldsymbol{\theta}\right)\right)_{T} \leq C h_{T}^{2}\left(\|\boldsymbol{\theta}\|_{H^{2}(T)}^{2}+\left\|u_{0}\right\|_{H^{3}(T)}^{2}\right) .
$$

By summation it thus follows that

$$
\left\|\boldsymbol{\theta}-\boldsymbol{\pi}_{u} \boldsymbol{\theta}\right\| \mid \leq C h\left(\|\boldsymbol{\theta}\|_{H^{2}(\Omega)}+\left\|u_{0}\right\|_{H^{3}(\Omega)}\right) .
$$


Finally, by the triangle inequality and standard interpolation arguments,

$$
\begin{aligned}
&\left\|\boldsymbol{\zeta}-\boldsymbol{Q}_{u} \boldsymbol{\zeta}\right\|_{L_{2}(\Omega)} \leq \| \boldsymbol{\zeta}-\boldsymbol{\pi}_{1} \boldsymbol{\zeta}\left\|_{L_{2}(\Omega)}+\frac{\kappa^{1 / 2}}{t^{2}}\right\| \nabla u_{r}-\nabla \pi_{2} u_{r} \|_{L_{2}(\Omega)} \\
&+\frac{\kappa^{1 / 2}}{t^{2}}\left\|\nabla u_{r}-\boldsymbol{\pi}_{1} \nabla u_{r}\right\|_{L_{2}(\Omega)} \\
& \leq C h\left(t^{-2}\left\|u_{r}\right\|_{H^{2}(\Omega)}+\|\boldsymbol{\zeta}\|_{H^{1}(\Omega)}\right)
\end{aligned}
$$

which completes the proof of the lemma.

We can now prove the following best approximation result.

Lemma 8 We have that

$$
\left\|\boldsymbol{\theta}-\boldsymbol{\theta}^{h} \mid\right\|+t\left\|\boldsymbol{\zeta}-\boldsymbol{\zeta}^{h}\right\|_{L_{2}(\Omega)} \leq C\left(\|\| \boldsymbol{\theta}-\boldsymbol{P}_{u} \boldsymbol{\theta}\|\mid+t\| \boldsymbol{\zeta}-\boldsymbol{Q}_{u} \boldsymbol{\zeta} \|_{L_{2}(\Omega)}\right) .
$$

PROOF. By the triangle inequality

$$
\begin{gathered}
\left\|\boldsymbol{\theta}-\boldsymbol{\theta}^{h}\right\| \mid+t\left\|\boldsymbol{\zeta}-\boldsymbol{\zeta}^{h}\right\|_{L_{2}(\Omega)} \leq \\
\|t\| \boldsymbol{\theta}-\boldsymbol{P}_{u} \boldsymbol{\theta}\left\|\left|+\left\|\boldsymbol{P}_{u} \boldsymbol{\theta}-\boldsymbol{\theta}^{h}\right\|\right|+t\right\| \boldsymbol{\zeta}-\boldsymbol{Q}_{u} \boldsymbol{\zeta} \|_{L_{2}(\Omega)} \\
+t\left\|\boldsymbol{Q}_{u} \boldsymbol{\zeta}-\boldsymbol{\zeta}^{h}\right\|_{L_{2}(\Omega)} .
\end{gathered}
$$

Further, by (17), we have that

$$
\begin{aligned}
\|\| \boldsymbol{\theta}^{h}- & \boldsymbol{P}_{u} \boldsymbol{\theta}\|\|^{2}+t^{2}\left\|\boldsymbol{\zeta}^{h}-\boldsymbol{Q}_{u} \boldsymbol{\zeta}\right\|_{L_{2}(\Omega)}^{2} \\
\leq & C\left(a_{h}\left(\boldsymbol{\theta}^{h}-\boldsymbol{P}_{u} \boldsymbol{\theta}, \boldsymbol{\theta}^{h}-\boldsymbol{P}_{u} \boldsymbol{\theta}\right)+t^{2}\left(\boldsymbol{\zeta}^{h}-\boldsymbol{Q}_{u} \boldsymbol{\zeta}, \boldsymbol{\zeta}^{h}-\boldsymbol{Q}_{u} \boldsymbol{\zeta}\right)\right) \\
= & C\left(a_{h}\left(\boldsymbol{\theta}-\boldsymbol{P}_{u} \boldsymbol{\theta}, \boldsymbol{\theta}^{h}-\boldsymbol{P}_{u} \boldsymbol{\theta}\right)+t^{2}\left(\boldsymbol{\zeta}-\boldsymbol{Q}_{u} \boldsymbol{\zeta}, \boldsymbol{\zeta}^{h}-\boldsymbol{Q}_{u} \boldsymbol{\zeta}\right)\right) \\
\leq & C\left(\left\|\boldsymbol{\theta}-\boldsymbol{P}_{u} \boldsymbol{\theta}\right\|+t\left\|\boldsymbol{\zeta}-\boldsymbol{Q}_{u} \boldsymbol{\zeta}\right\|_{L_{2}(\Omega)}\right) \\
& \times\left(\left\|\boldsymbol{\theta}^{h}-\boldsymbol{P}_{u} \boldsymbol{\theta}\right\|+t\left\|\boldsymbol{\zeta}^{h}-\boldsymbol{Q}_{u} \boldsymbol{\zeta}\right\|_{L_{2}(\Omega)}\right),
\end{aligned}
$$

and the lemma follows.

Finally, combining Lemmas 6, 7, and 8, we obtain

Theorem 9 If $\Omega$ is a convex domain and $g \in L_{2}(\Omega)$ we have, for $\left(\boldsymbol{\theta}^{h}, u^{h}\right)$ solving (6) and $(\boldsymbol{\theta}, u)$ solving (4), and using the definition (16),

$$
\left\|\boldsymbol{\theta}-\boldsymbol{\theta}^{h}\right\| \mid+t\left\|\boldsymbol{\zeta}-\boldsymbol{\zeta}^{h}\right\|_{L_{2}(\Omega)} \leq C h\left(\|g\|_{H^{-1}(\Omega)}+t\|g\|_{L_{2}(\Omega)}\right),
$$

uniformly in $t$. 


\subsection{An estimate in $L_{2}$-norm}

Consider the following auxiliary problem of finding $z$ and $\psi$ such that

$$
\begin{aligned}
-\nabla \cdot \boldsymbol{\sigma}(\boldsymbol{\psi})-\kappa t^{-2}(\nabla z-\boldsymbol{\psi})=\boldsymbol{\theta}-\boldsymbol{\theta}^{h}, & \text { in } \Omega, \\
-\kappa t^{-2} \nabla \cdot(\nabla z-\boldsymbol{\psi})=u-u^{h}, & \text { in } \Omega,
\end{aligned}
$$

which, with $e_{u}=u-u^{h}$ and $\boldsymbol{e}_{\boldsymbol{\theta}}=\boldsymbol{\theta}-\boldsymbol{\theta}^{h}$, leads to

$$
\left\|e_{u}\right\|_{L_{2}(\Omega)}^{2}+\left\|\boldsymbol{e}_{\boldsymbol{\theta}}\right\|_{L_{2}(\Omega)}^{2}=a_{h}\left(\boldsymbol{e}_{\boldsymbol{\theta}}, \boldsymbol{\psi}\right)+b\left(e_{u}, \boldsymbol{e}_{\boldsymbol{\theta}} ; z, \boldsymbol{\psi}\right)
$$

using integration by parts and the symmetry of the forms. Proceeding as in the energy estimate, we define

$$
\boldsymbol{\eta}:=\kappa^{1 / 2}(\nabla z-\boldsymbol{\psi}) / t^{2}
$$

and the split $z=z_{0}+z_{r}$. We also define $\boldsymbol{e}_{\boldsymbol{\zeta}}=\boldsymbol{\zeta}-\boldsymbol{\zeta}^{h}$ and introduce the operators $\boldsymbol{Q}_{z}$ and $\boldsymbol{P}_{z}$ defined as in Lemma 6 (with $z$ in the place of $u$ and $\boldsymbol{\psi}$ in the place of $\boldsymbol{\theta})$. Then, by Lemma 2, we find that

$$
\begin{aligned}
\left\|e_{u}\right\|_{L_{2}(\Omega)}^{2}+\left\|\boldsymbol{e}_{\boldsymbol{\theta}}\right\|_{L_{2}(\Omega)}^{2}= & a_{h}\left(\boldsymbol{e}_{\boldsymbol{\theta}}, \boldsymbol{\psi}-\boldsymbol{P}_{z} \boldsymbol{\psi}\right)+t^{2}\left(\boldsymbol{e}_{\boldsymbol{\zeta}}, \boldsymbol{\eta}-\boldsymbol{Q}_{z} \boldsymbol{\eta}\right) \\
\leq C & \left(\left\|\boldsymbol{e}_{\boldsymbol{\theta}}\right\| \mid+t\left\|\boldsymbol{e}_{\boldsymbol{\zeta}}\right\|_{L_{2}(\Omega)}\right) \\
& \times\left(\left\|\boldsymbol{\psi}-\boldsymbol{P}_{z} \boldsymbol{\psi}\right\|+t\left\|\boldsymbol{\eta}-\boldsymbol{Q}_{z} \boldsymbol{\eta}\right\|_{L_{2}(\Omega)}\right) .
\end{aligned}
$$

Proceeding as in Lemma 7, and using the stability result

$$
\left\|z_{0}\right\|_{H^{3}(\Omega)}+\frac{1}{t}\left\|z_{r}\right\|_{H^{2}(\Omega)}+\|\boldsymbol{\psi}\|_{H^{2}(\Omega)}+t\|\boldsymbol{\eta}\|_{H^{1}(\Omega)} \leq C\left(\left\|e_{u}\right\|_{L_{2}(\Omega)}+\left\|\boldsymbol{e}_{\boldsymbol{\theta}}\right\|_{L_{2}(\Omega)}\right) .
$$

adapted from [7,1], we finally obtain (under the conditions of Theorem 9) the $a$ priori estimate:

$$
\left\|u-u^{h}\right\|_{L_{2}(\Omega)}+\left\|\boldsymbol{\theta}-\boldsymbol{\theta}^{h}\right\|_{L_{2}(\Omega)} \leq C h^{2}\left(\|g\|_{H^{-1}(\Omega)}+t\|g\|_{L_{2}(\Omega)}\right) .
$$




\section{A posteriori error estimates and adaptive algorithms}

\subsection{A duality-based a posteriori estimate}

For duality-based a posteriori error analysis, we consider the following variant of (19): find $z$ and $\psi$ such that

$$
\begin{aligned}
-\nabla \cdot \boldsymbol{\sigma}(\boldsymbol{\psi})-\kappa t^{-2}(\nabla z-\boldsymbol{\psi}) & =\boldsymbol{f}_{\theta}, \quad \text { in } \Omega \\
-\kappa t^{-2} \nabla \cdot(\nabla z-\boldsymbol{\psi}) & =f_{u}, \quad \text { in } \Omega
\end{aligned}
$$

with zero Dirichlet boundary conditions for $z$ and $\boldsymbol{\psi}$. With $\boldsymbol{e}_{\theta}=\boldsymbol{\theta}-\boldsymbol{\theta}^{h}$ and $e_{u}=$ $u-u^{h}$ we find, using Lemma 2 , that

$$
\begin{aligned}
\left(\boldsymbol{f}_{\theta}, \boldsymbol{e}_{\theta}\right)_{\Omega}+\left(f_{u}, e_{u}\right)_{\Omega} & =a_{h}\left(\boldsymbol{\psi}, \boldsymbol{e}_{\theta}\right)+b\left(z, \boldsymbol{\psi} ; e_{u}, \boldsymbol{e}_{\theta}\right) \\
& =a_{h}\left(\boldsymbol{e}_{\theta}, \boldsymbol{\psi}-\pi_{h} \boldsymbol{\psi}\right)+b\left(e_{u}, \boldsymbol{e}_{\theta} ; z-\tilde{\pi}_{h} z, \boldsymbol{\psi}-\pi_{h} \boldsymbol{\psi}\right)
\end{aligned}
$$

where $\pi_{h}$ and $\tilde{\pi}_{h}$ now denote arbitrary interpolants (or projections) onto the respective subspaces. Using the equilibrium equations we find that

$$
\begin{aligned}
\left(\boldsymbol{f}_{\theta}, \boldsymbol{e}_{\theta}\right)_{\Omega}+\left(f_{u}, e_{u}\right)_{\Omega}= & \left(g, z-\tilde{\pi}_{h} z\right)-a_{h}\left(\boldsymbol{\theta}^{h}, \boldsymbol{\psi}-\pi_{h} \boldsymbol{\psi}\right) \\
& -b\left(u^{h}, \boldsymbol{\theta}^{h} ; z-\tilde{\pi}_{h} z, \boldsymbol{\psi}-\pi_{h} \boldsymbol{\psi}\right) .
\end{aligned}
$$

This exact relation forms the basis of our adaptive error control algorithm. Following Becker and Rannacher [3], we can now select the terms $\boldsymbol{f}_{\theta}$ and $f_{u}$ to try to control general linear functionals of the errors in displacement and rotation, as long as we have good estimates of the a priori terms $z-\tilde{\pi}_{h} z$ and $\psi-\pi_{h} \psi$.

\subsection{Implementation}

We shall establish a practical adaptive method based on (23). To this end we need to approximate the (unknown) solution of the continuous dual plate problem (22). In the numerical examples presented in Sections 7.1-7.2, we aim at controlling errors in displacements, which implies $\boldsymbol{f}_{\theta}=\mathbf{0}$, and the discretized dual plate formulation becomes: find $\tilde{z} \in V_{h}^{*}$ and $\tilde{\boldsymbol{\psi}} \in \Theta_{h}^{*}$ such that

$$
a_{h}(\tilde{\boldsymbol{\psi}}, \boldsymbol{\vartheta})+b(\tilde{z}, \tilde{\boldsymbol{\psi}} ; v, \boldsymbol{\vartheta})=\left(f_{u}, v\right)_{\Omega}, \quad \forall(v, \boldsymbol{\vartheta}) \in V_{h}^{*} \times \boldsymbol{\Theta}_{h}^{*},
$$

where the enriched function spaces, $V_{h}^{*} \supset V_{h}$ and $\Theta_{h}^{*} \supset \Theta_{h}$, are constructed by regular subdivision of the primal triangulation $\mathfrak{T}$. For simplicity we let $k=2$, with quadratic and linear approximations of the transverse displacements and the rotation vector, respectively. 
The positive constant $\gamma$ in $a_{h}(\cdot, \cdot)$ must be large enough to ensure coercivity, cf. Lemma 4. A discussion on how to compute the stability parameter, in the context of the Kirchhoff plate model, can be found in [11]. Typically $\gamma$ increases with the order of the polynomial approximation. Here we set $\gamma=10$, which is larger than the asymptotic value, $\gamma_{0}=3 / 2$. We also note that the presence of the shear energy functional (3) in the Mindlin-Reissner model stabilizes the numerical method (6) further. The implications of the choice of stability parameter on different types of meshes are discussed in Section 7.4.

Now, after solving (24) the error estimate (23) is evaluated elementwise, so that each $T_{j} \in \mathfrak{T}, j=1, \ldots, N$, contributes $\eta_{j}$ to the total error

$$
L\left(e_{u}\right)=\left(f_{u}, e_{u}\right)_{\Omega} \approx \sum_{j=1}^{N} \eta_{j}=L\left(\tilde{e}_{u}\right) .
$$

Error contributions in (23) coming from internal edges are split equally between neighboring elements. Moreover, $\tilde{\pi}_{h}: V_{h}^{*} \rightarrow V_{h}$ is chosen to be the nodal interpolant of $\tilde{z}$, whereas $\pi_{h}: \Theta_{h}^{*} \rightarrow \Theta_{h}$ is an elementwise $L_{2}$-projection of the rotation components. The stopping criterion of the adaptive algorithm, summarized in Algorithm 1, is imposed on the relative error

$$
e_{\mathrm{rel}}:=\left|L\left(e_{u}\right) / L(u)\right| \leq \mathrm{TOL}
$$

where TOL $>0$ is a prescribed tolerance. If (25) is not satisfied, a fixed-ratio, here chosen as $r=20 \%$, of the elements with the largest absolute error indicators $\left|\eta_{j}\right|$ are locally refined using longest-edge bisection. The performance of the $a$ posteriori error estimator is evaluated in terms of the effectivity index

$$
I_{\mathrm{eff}}:=\left|L\left(\tilde{e}_{u}\right) / L\left(e_{u}\right)\right| .
$$

When the computational mesh is sufficiently resolved, we want the estimator to be robust and accurate. The ultimate goal is to have $I_{\text {eff }}=1$ exactly; however, since we use numerical approximations of the continuous dual plate problem, this cannot be expected and we observe a slight overestimation of the error.

\section{Numerical examples}

We apply Algorithm 1 to a set of simpler model problems in order to: 1) exemplify the behavior of the adaptive procedure; 2) study convergence rates of the finite element method (6) with respect to meshsize and plate thickness; and 3) study how the choice of stability parameter affects the approximation on different types of meshes. 


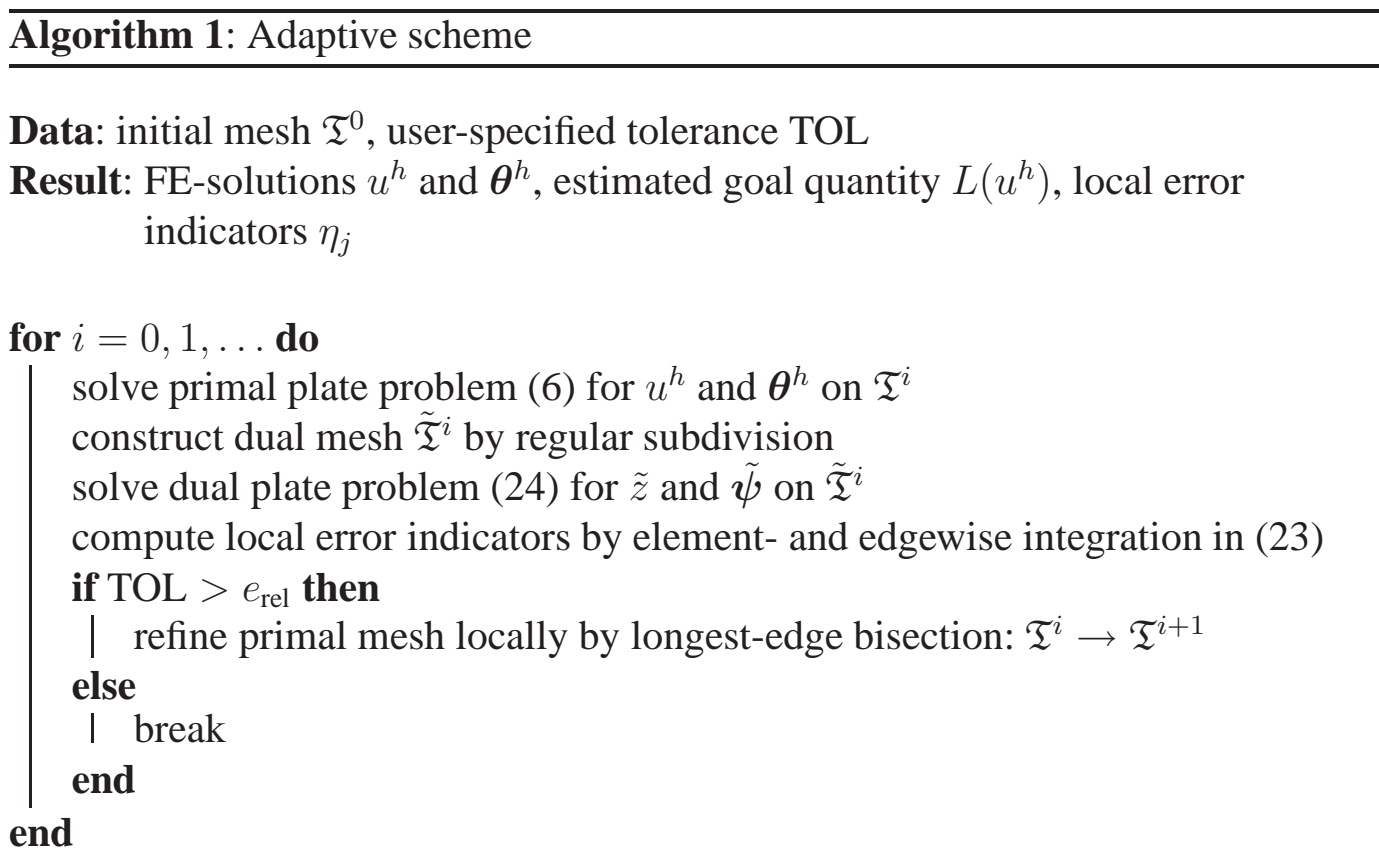

The stopping criteria were set to require a reasonable number of refinement levels, given initial meshes $\mathfrak{T}^{0}$ not too coarse. The shear correction factor $k=5 / 6$.

We remark that the solutions in Sections 7.1 and 7.2 are not smooth enough to be covered by the a priori error analysis presented above. We chose these examples in order to show that the adaptive algorithm still can predict the error accurately. In Section 7.3 we show the convergence in a smooth case.

\subsection{An L-shaped membrane}

The polygonal domain $\Omega$, with vertices at $(0,0),\left(\frac{1}{2}, 0\right),\left(\frac{1}{2}, \frac{1}{2}\right),\left(1, \frac{1}{2}\right),(1,1)$, and $(0,1)$, represents a clamped plate $\left(\left.u\right|_{\partial \Omega}=0\right.$ and $\left.\left.\boldsymbol{\theta}\right|_{\partial \Omega}=0\right)$. The material parameters $\nu=1 / 3, E=1$, and the thickness $t=10^{-2}$. The plate is subjected to a uniform transverse load $g=1$.

We set $f_{u}=1$ to control the error in mean displacement. The exact goal quantity was approximated by

$$
L(u)=\int_{\Omega} u \mathrm{~d} \Omega \approx \int_{\Omega} u^{\mathrm{app}} \mathrm{d} \Omega,
$$

where $u^{\text {app }}$ denotes the approximate solution on a densely adapted mesh. Hence the effectivity index can be expected to increase slowly during the adaptive process. Due to the symmetry of the domain and the uniform load, consecutive meshes should also be symmetric. A concentration of elements is expected in the vicinity of the corner singularity at $\left(\frac{1}{2}, \frac{1}{2}\right)$. 
The FE-solution $u^{h}$, shown in Figure 1, was computed on the nearly symmetric (about $y=1-x$ ) final mesh $\mathfrak{T}^{10}$, shown in Figure 2(b). Local refinements were prominent at the interior corner, and along the boundaries of the domain, where the magnitude of the curvature of the plate becomes large. The initial mesh is shown in Figure 2(a). The effectivity indices shown in Figure 3(b) indicate the accurate and robustness of the error estimator. The adaptive strategy is more efficient in terms of accuracy per degree of freedom compared to uniform refinement according to the results presented in Figure 3(a). Data from the adaptive process are summarized in Table 1.

\subsection{The unit square}

Here, we let $\Omega=[0,1] \times[0,1]$ represent a fixed plate $\left(\left.\boldsymbol{\theta}\right|_{\partial \Omega}=0\right)$. We use the material parameters and thickness from the preceding example, i.e., $E=1, \nu=1 / 3$, and $t=10^{-2}$. The rotation vector is given explicitly by

$$
\boldsymbol{\theta}=\left[\begin{array}{l}
2(x-1) x(2 x-1)(y-1)^{2} y^{2} \\
2(y-1) y(2 y-1)(x-1)^{2} x^{2}
\end{array}\right],
$$

and the symmetric surface load

$$
\begin{aligned}
g(x, y)= & \frac{2 E}{1-\nu^{2}}\left(x(x-1)\left(x^{2}-x+2\right)+y(y-1)\left(y^{2}-y+2\right)\right. \\
& \left.+12 x(x-1) y(y-1)+\frac{1}{3}\right) .
\end{aligned}
$$

This corresponds to the transverse displacements

$$
\begin{aligned}
u(x, y)= & \frac{t^{2}}{5(\nu-1)}\left(12 x(x-1) y(y-1)\left(x^{2}-x+y^{2}-y\right)\right. \\
& \left.+2\left(x^{2}(x-1)^{2}+y^{2}(y-1)^{2}\right)\right)+x^{2}(x-1)^{2} y^{2}(y-1)^{2},
\end{aligned}
$$

whose boundary values are applied as Dirichlet data. Note that the displacements will depend on the thickness of the plate, so that we regain the Kirchhoff solution, $u_{0}(x, y)=x^{2}(x-1)^{2} y^{2}(y-1)^{2}$, in the limit $t \rightarrow 0$.

We let the datum of the dual plate problem be a Dirac delta function

$$
f_{u}=\delta(x-\bar{x}, y-\bar{y}), \quad(\bar{x}, \bar{y})=\left(\frac{3}{4}, \frac{3}{4}\right),
$$

so that the error in the goal quantity simplifies to $L\left(e_{u}\right)=u(\bar{x}, \bar{y})-u^{h}(\bar{x}, \bar{y})$ corresponding to controlling the displacement error in a single point. Since the analytical solution is symmetric, we expect consecutive meshes to be symmetric as well, with respect to the symmetry line $y=x$. The final mesh should be densely resolved about $(\bar{x}, \bar{y})$. 
The FE-solution shown in Figure 5, was computed on the nearly symmetric final mesh $\mathfrak{T}^{11}$, given in Figure 4(b). The initial mesh, shown in Figure 4(a), was refined by the algorithm, in particular in the vicinity of $(\bar{x}, \bar{y})$, but also along the boundary. Figure $6(\mathrm{~b})$ shows the effectivity index to be stable, with $L\left(\tilde{e}_{u}\right)$ slightly overestimating $L\left(e_{u}\right)$. The comparison presented in Figure 6(a) indicates that the adaptive strategy is more efficient than uniform refinement. The absence of a strong singularity, however, makes it less so than in the previous example. Numerical data are given in Table 2.

\subsection{Convergence in meshsize and plate thickness}

We now consider convergence rates $\alpha$ of the numerical solution in terms of meshsize and plate thickness. To this end, the model problem in Section 7.2, with known analytical solutions (27) and (28), will be employed.

For the displacements the result in Figure 7(a) indicates the optimal rate of secondorder convergence in $L_{2}$-norm, which is in agreement with the order of the polynomial approximation. The same result can be seen in Figure 7(b), asymptotically as $h \rightarrow 0$, for the scaled shear stresses (16). These numerical convergence rates concur with (21). For the rotations, in Figure 8(a), we observe first-order convergence in the broken $H^{1}$-norm, which, by Lemma 3, is in accordance with the error estimate of Theorem 9. The latter is also confirmed by the results in Figure 8(b). Moreover, Theorem 9 predicts uniform convergence in $t$, which is shown in Figure 9 for a set of different plate thicknesses, ranging from $10^{-1}$ to $10^{-6}$.

Finally, in order to avoid shear locking, the difference $\nabla u-\boldsymbol{\theta}$ must vanish in the limit $t \rightarrow 0$. The results in Figure 10 show that $\left\|\nabla u^{h}-\boldsymbol{\theta}^{h}\right\|_{L_{2}(\Omega)}$ converges to zero, almost quadratically, as $t \rightarrow 0$ for fixed $h$ (as in the analytical solution). Hence the computed shear energy $\frac{1}{2} b\left(u^{h}, \boldsymbol{\theta}^{h} ; u^{h}, \boldsymbol{\theta}^{h}\right)$ does as well.

\subsection{Choice of stability parameter}

We study the sensitivity of the numerical solution to the choice of stability parameter $\gamma$. This is done on three different types of meshes: 1) a mesh with directionality; 2) an unstructured mesh; and 3) a criss-cross mesh. We reuse our model problem in Section 7.2, and consider the error in the midpoint displacement, as a function of $\gamma$ and plate thickness. The different meshes were constructed to have a similar number of elements.

The numerical method (6) has a mesh sensitivity with respect to $\gamma$ for low-order $k$, as discussed in [11], in context of the Kirchhoff plate model. If $\gamma$ is chosen too large locking will occur, unless a global $C^{1}$-approximation is contained in the trial space. 
Table 1

Goal-oriented adaptive procedure: controlling the error in mean displacements on an Lshaped membrane

\begin{tabular}{rrrrrrc}
\hline iter. & \# elements & \# DOF & goal quantity & est. error & est. exact error & effectivity \\
\hline 1 & 384 & 3137 & $5.18 \cdot 10^{-4}$ & $8.08 \cdot 10^{-5}$ & $8.62 \cdot 10^{-5}$ & 0.93 \\
2 & 492 & 4001 & $5.44 \cdot 10^{-4}$ & $5.92 \cdot 10^{-5}$ & $5.93 \cdot 10^{-5}$ & 0.99 \\
3 & 604 & 4899 & $5.64 \cdot 10^{-4}$ & $4.03 \cdot 10^{-5}$ & $3.98 \cdot 10^{-5}$ & 1.01 \\
4 & 830 & 6737 & $5.75 \cdot 10^{-4}$ & $3.14 \cdot 10^{-5}$ & $2.92 \cdot 10^{-5}$ & 1.07 \\
5 & 1090 & 8831 & $5.84 \cdot 10^{-4}$ & $2.20 \cdot 10^{-5}$ & $2.01 \cdot 10^{-5}$ & 1.09 \\
6 & 1422 & 11499 & $5.90 \cdot 10^{-4}$ & $1.53 \cdot 10^{-5}$ & $1.38 \cdot 10^{-5}$ & 1.11 \\
7 & 1954 & 15803 & $5.94 \cdot 10^{-4}$ & $1.15 \cdot 10^{-5}$ & $1.01 \cdot 10^{-5}$ & 1.14 \\
8 & 2686 & 21699 & $5.96 \cdot 10^{-4}$ & $8.67 \cdot 10^{-6}$ & $7.40 \cdot 10^{-6}$ & 1.17 \\
9 & 3567 & 28760 & $5.98 \cdot 10^{-4}$ & $6.37 \cdot 10^{-6}$ & $5.30 \cdot 10^{-6}$ & 1.20 \\
10 & 4740 & 38179 & $6.00 \cdot 10^{-4}$ & $4.76 \cdot 10^{-6}$ & $3.83 \cdot 10^{-6}$ & 1.24 \\
\hline
\end{tabular}

Table 2

Goal-oriented adaptive procedure: controlling the displacement error in a point on the unit square

\begin{tabular}{rrrrrrr}
\hline iter. & \# elements & \# DOF & goal quantity & est. error & exact error & effectivity \\
\hline 1 & 512 & 4161 & $1.1857 \cdot 10^{-3}$ & $5.49 \cdot 10^{-5}$ & $5.07 \cdot 10^{-5}$ & 1.08 \\
2 & 654 & 5297 & $1.2062 \cdot 10^{-3}$ & $3.36 \cdot 10^{-5}$ & $3.02 \cdot 10^{-5}$ & 1.11 \\
3 & 840 & 6793 & $1.2159 \cdot 10^{-3}$ & $2.26 \cdot 10^{-5}$ & $2.04 \cdot 10^{-5}$ & 1.10 \\
4 & 1122 & 9061 & $1.2216 \cdot 10^{-3}$ & $1.70 \cdot 10^{-5}$ & $1.48 \cdot 10^{-5}$ & 1.14 \\
5 & 1468 & 11831 & $1.2248 \cdot 10^{-3}$ & $1.34 \cdot 10^{-5}$ & $1.16 \cdot 10^{-5}$ & 1.15 \\
6 & 1996 & 16093 & $1.2277 \cdot 10^{-3}$ & $1.00 \cdot 10^{-5}$ & $8.70 \cdot 10^{-6}$ & 1.15 \\
7 & 2672 & 21515 & $1.2302 \cdot 10^{-3}$ & $7.29 \cdot 10^{-6}$ & $6.26 \cdot 10^{-6}$ & 1.16 \\
8 & 3604 & 28995 & $1.2318 \cdot 10^{-3}$ & $5.43 \cdot 10^{-6}$ & $4.61 \cdot 10^{-6}$ & 1.17 \\
9 & 4810 & 38675 & $1.2331 \cdot 10^{-3}$ & $3.85 \cdot 10^{-6}$ & $3.30 \cdot 10^{-6}$ & 1.16 \\
10 & 6492 & 52177 & $1.2341 \cdot 10^{-3}$ & $2.76 \cdot 10^{-6}$ & $2.35 \cdot 10^{-6}$ & 1.17 \\
11 & 8662 & 69561 & $1.2346 \cdot 10^{-3}$ & $2.13 \cdot 10^{-6}$ & $1.79 \cdot 10^{-6}$ & 1.18 \\
\hline
\end{tabular}


The effect is evident for thin plates, as seen in Figures 11 and 12, where the degradation of the numerical solutions on oriented and unstructured meshes is shown. On a criss-cross mesh, however, the finite element method is robust with respect to locking: the existence of a $C^{1}$-approximation with second degree polynomials on this type of mesh was shown by Zhang in [16]. In Figure 13 we also note how $u \rightarrow u_{0}$ as $t \rightarrow 0$. Finally, we remark that there is no numerical locking for thicker plates; here $\gamma$ can be set arbitrarily large (though this will affect the accuracy on a fixed mesh).

\section{CONCLUDING REMARKS}

We have presented a novel finite element method for the Mindlin-Reissner plate model, based on the discontinuous Galerkin approach. We show that our method does not lock as long as we make a proper choice of a free, but computable, parameter. Our approach avoids the current paradigm of projections of the rotations in the shear energy functional, which, at least from a conceptual point of view, requires a mixed implementation. We pay the price of having to use a higher number of degrees of freedom; in consequence, the presented approach may not be computationally competitive with the "best" elements available. Nevertheless, we feel that it is a very simple and straightforward method; in particular it is free of special mixed element approximations.

\section{References}

[1] D. N. Arnold, R. S. Falk, A uniformly accurate finite-element method for the ReissnerMindlin plate, SIAM Journal of Numerical Analysis 26 (1989) 1276-1290

[2] K. J. Bathe, E. N. Dvorkin, A four-node plate bending element based on MindlinReissner plate theory and mixed interpolation, International Journal for Numerical Methods in Engineering 21 (1985) 367-383.

[3] R. Becker and R. Rannacher, A feed-back approach to error control in finite element methods: basic analysis and examples, East-West Journal of Numerical Mathematics 4 (1996) 237-264.

[4] P Bösing, A. L. Madureira, I. Mozolevski, A new interior penalty discontinuous Galerkin method for the Reissner-Mindlin model, Mathematical Models and Methods in Applied Sciences 20 (2010) 1343-1361.

[5] S. C. Brenner, Korn's inequalities for piecewise $H^{1}$ vector fields, Mathematics of Computation 73 (2004) 1067-1087. 
[6] G. Brezzi, M. Fortin, R. Stenberg, Error analysis of mixed-interpolated elements for Reissner-Mindlin plates, Mathematical Models and Methods in Applied Sciences 1 (1991) 125-151

[7] D. Chapelle, R. Stenberg, An optimal low-order locking-free finite element method for Reissner-Mindlin plates, Mathematical Models and Methods in Applied Sciences 8 (1998) 407-430

[8] R. Duran, E. Liberman, On mixed finite-element methods for the Reissner-Mindlin plate model, Mathematics of Compututation 58 (1992) 561-573.

[9] G. Engel, K. Garikipati, T. J. R. Hughes, M. G. Larson, L. Mazzei, R. L. Taylor, Continuous/discontinuous approximations of fourth-order elliptic problems in structural and continuum mechanics with applications to thin bending elements and strain gradient elasticity, Computer Methods in Applied Mechanics and Engineering 191 (2002) 3669-3750

[10] P. Hansbo, P. M. G. Larson, A $P^{2}$-continuous, $P^{1}$-discontinuous Galerkin method for the Mindlin-Reissner plate model, in: Numerical Mathematics and Advanced Applications: ENUMATH 2001, F. Brezzi, A. Buffa, S. Corsaro, and A. Murli (Eds.), Springer, Milan, 2003, pp. 765-774.

[11] P. Hansbo, M. G. Larson, A posteriori error estimates for continuous/discontinuous Galerkin approximations of the Kirchhoff-Love plate, Preprint 2008:10, Department of Mathematical Sciences, Chalmers University of Technology, 2008.

[12] P. Hansbo, M. G. Larson, A discontinuous Galerkin method for the plate problem, Calcolo 39 (2002) 41-59.

[13] P. Hansbo, M. G. Larson, Discontinuous Galerkin methods for incompressible and nearly incompressible elasticity by Nitsche's method, Computer Methods in Applied Mechanics and Engineering 191 (2002) 1895-1908

[14] J. Pitkäranta, Analysis of some low-order finite-element schemes for Mindlin-Reissner and Kirchhoff plates, Numerische Mathematik 53 (1988) 237-254

[15] Thomée V. Galerkin Finite Element Methods for Parabolic Problems. Springer-Verlag, Berlin, 1977

[16] Zhang S. A $C^{1}-P^{2}$ finite element without nodal basis, ESAIM: Mathematical Modelling and Numerical Analysis 42 (2008) 172-192. 


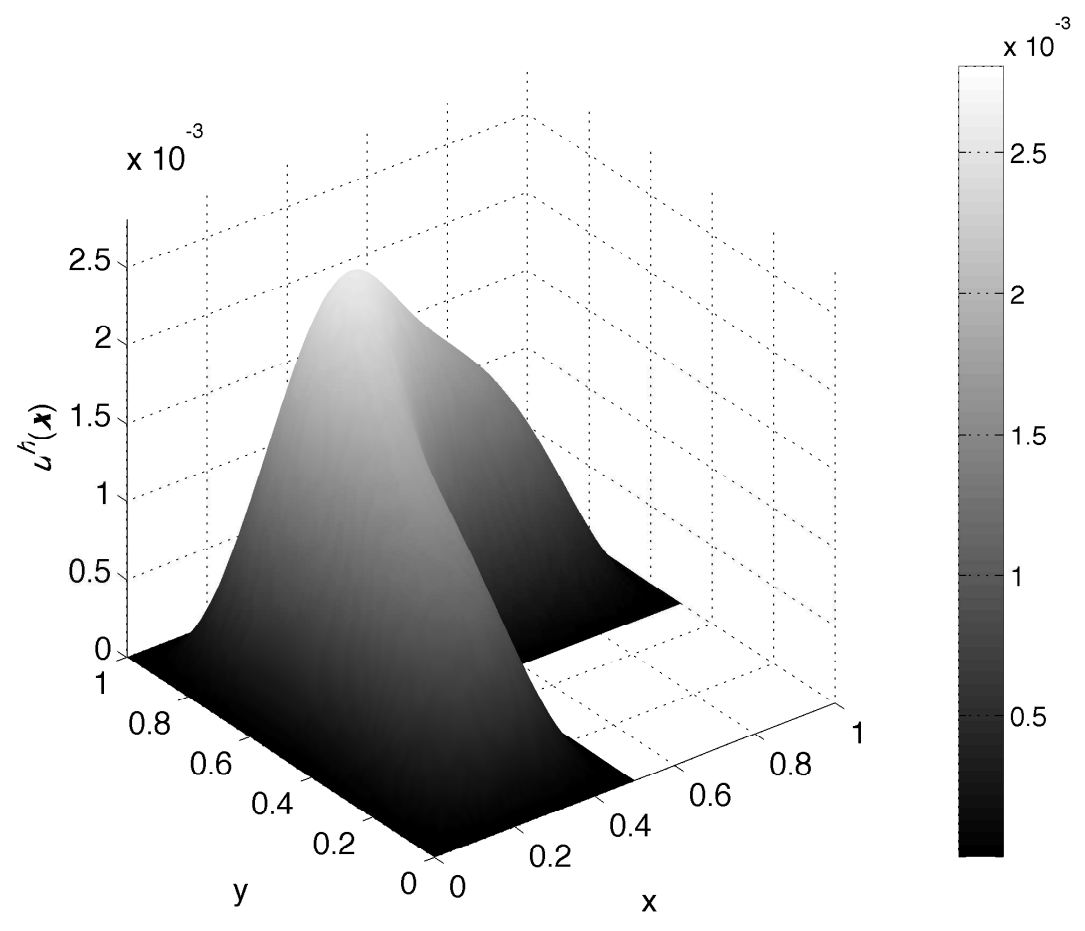

Fig. 1. The adapted FE-solution $u^{h}$ at the last refinement level $i=10$

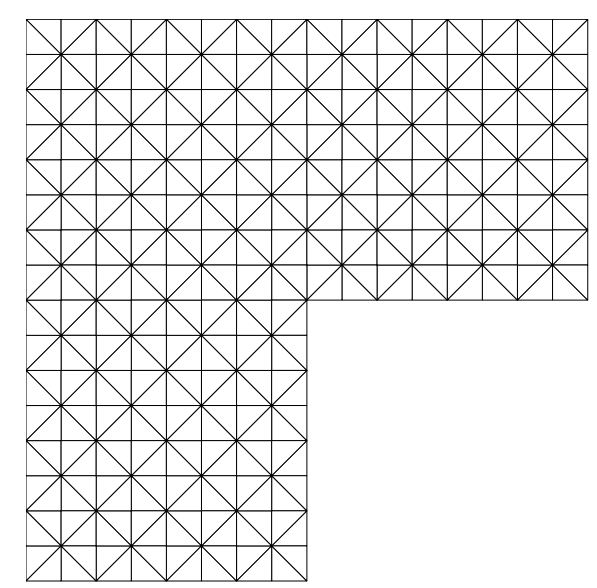

(a) Initial mesh $\mathfrak{T}^{0}$

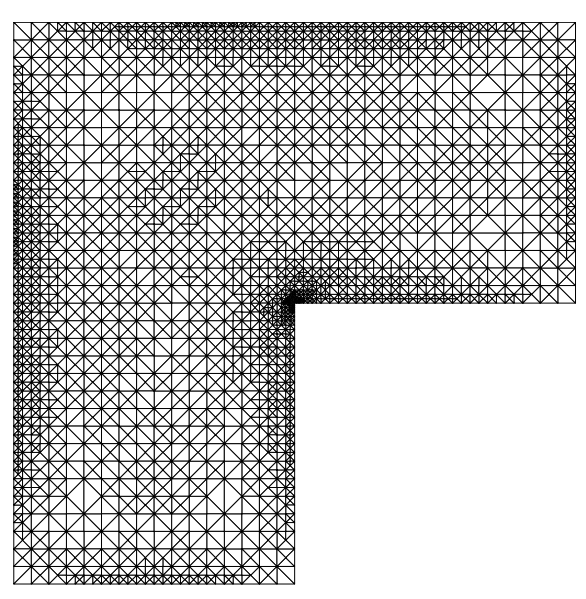

(b) Final mesh $\mathfrak{T}^{10}$

Fig. 2. 


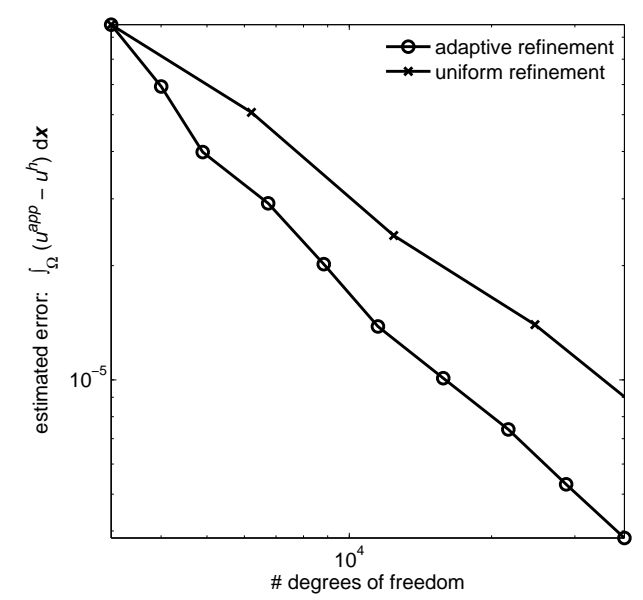

(a) Estimated error in the goal quantity $L\left(e_{u}\right)$, the mean displacement, at each refinement level.

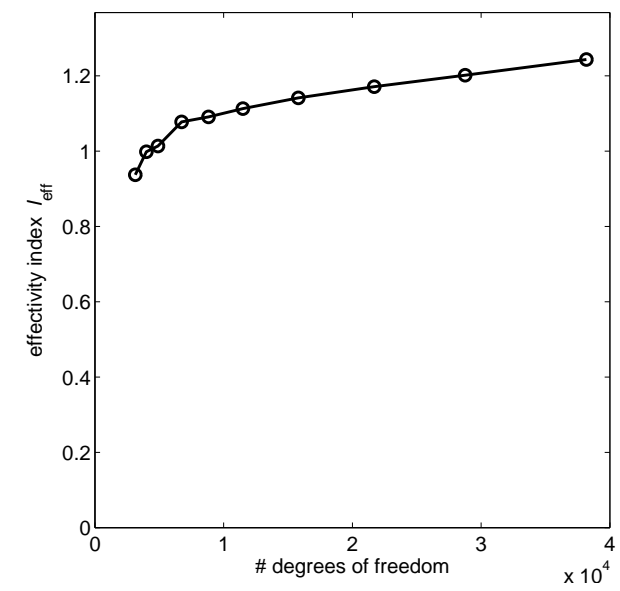

(b) Estimated effectivity index $I_{\text {eff }}$ at each refinement level.

Fig. 3.

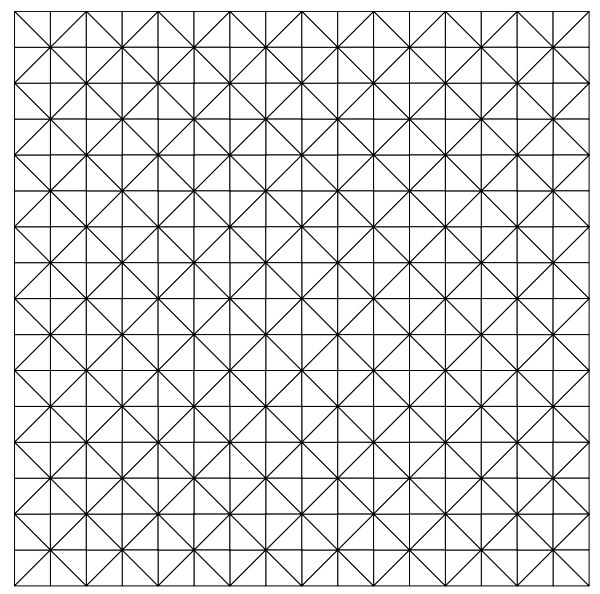

(a) Initial mesh $\mathfrak{T}^{0}$

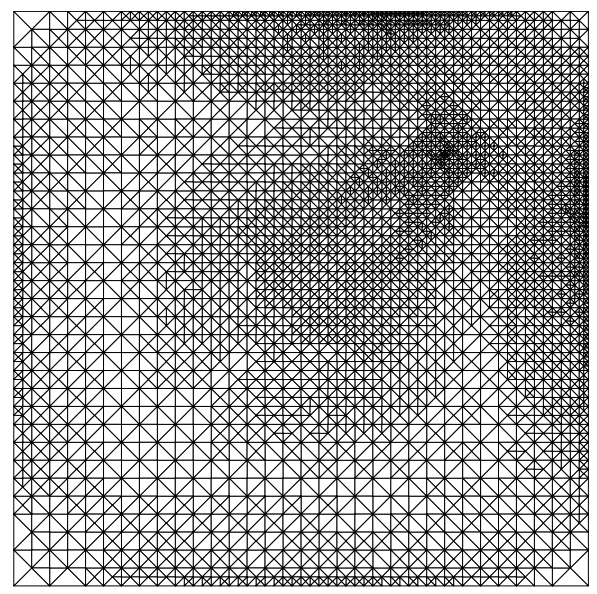

(b) Final mesh $\mathfrak{T}^{11}$

Fig. 4. 


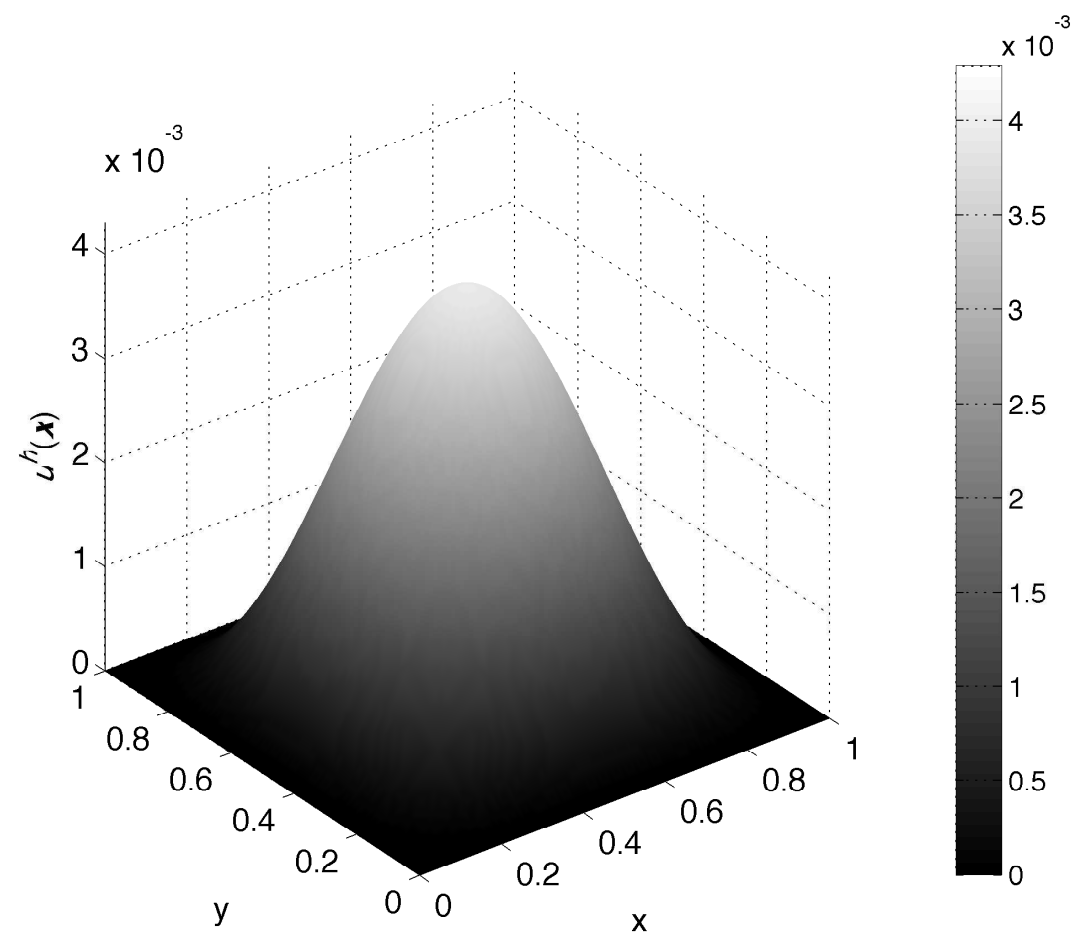

Fig. 5. The adapted FE-solution $u^{h}$ at the last refinement level $i=11$

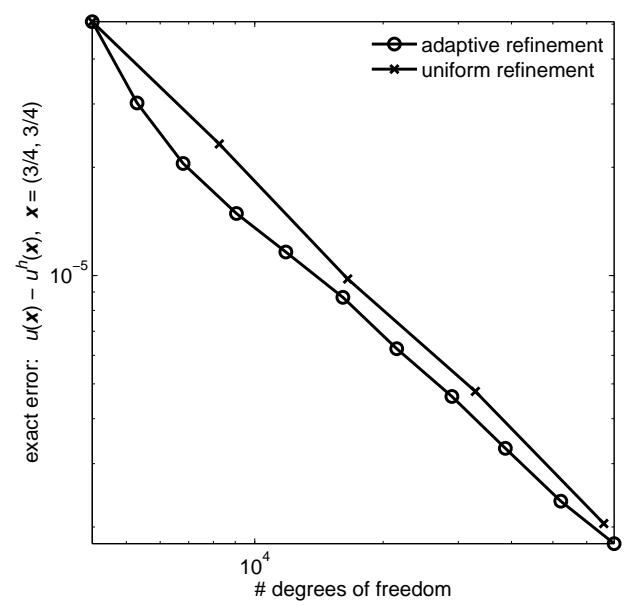

(a) Exact error in the goal quantity $L\left(e_{u}\right)$, a single point displacement, at each refinement level.

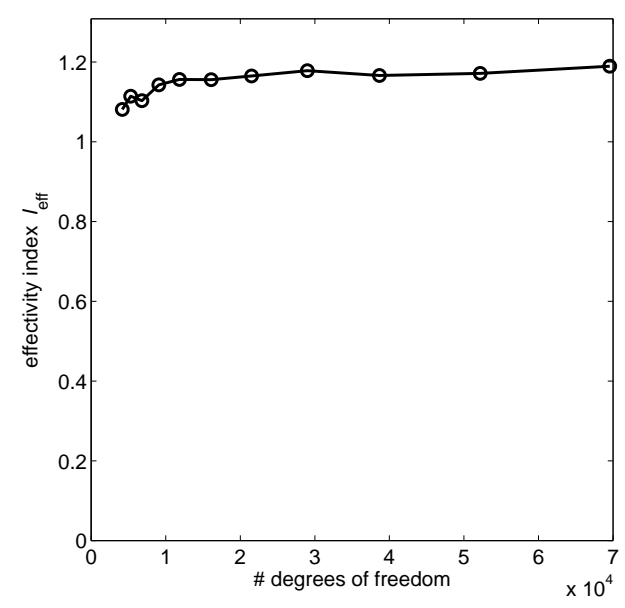

(b) Effectivity index $I_{\text {eff }}$ at each refinement level.

Fig. 6. 


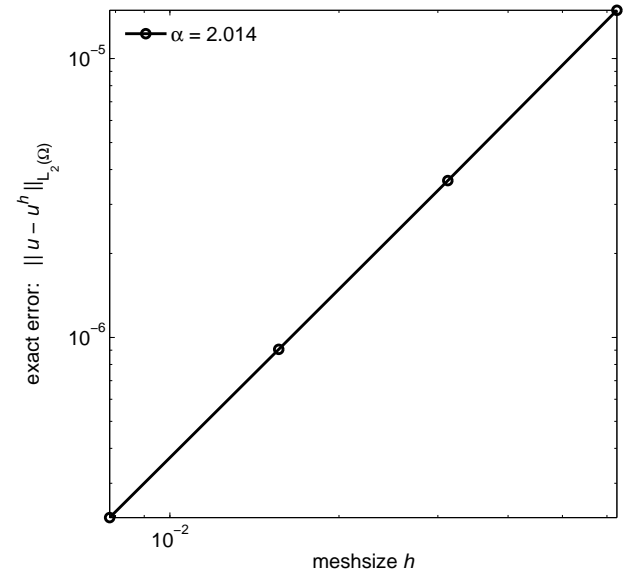

(a) Error in displacements as a function of meshsize.

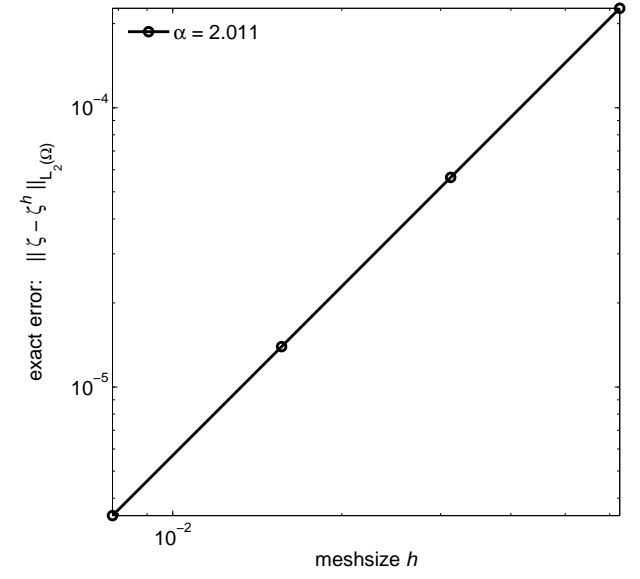

(b) Error in scaled shear stresses as a function of meshsize.

Fig. 7.

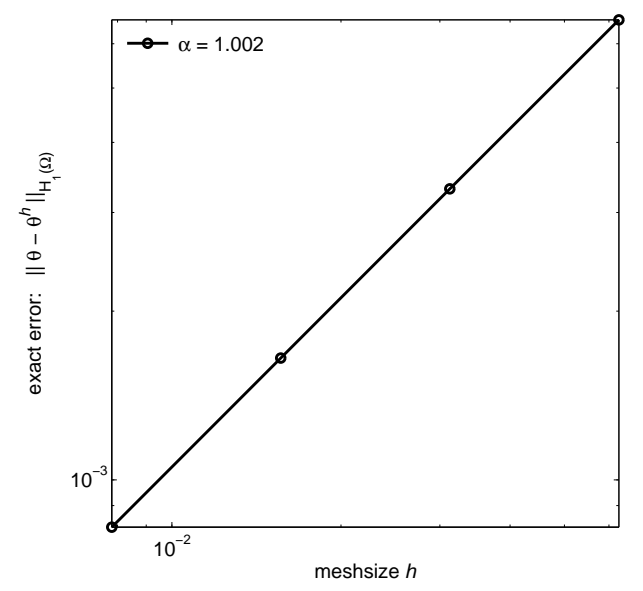

(a) Error in rotations, in broken $H^{1}$-norm, as a function of meshsize.

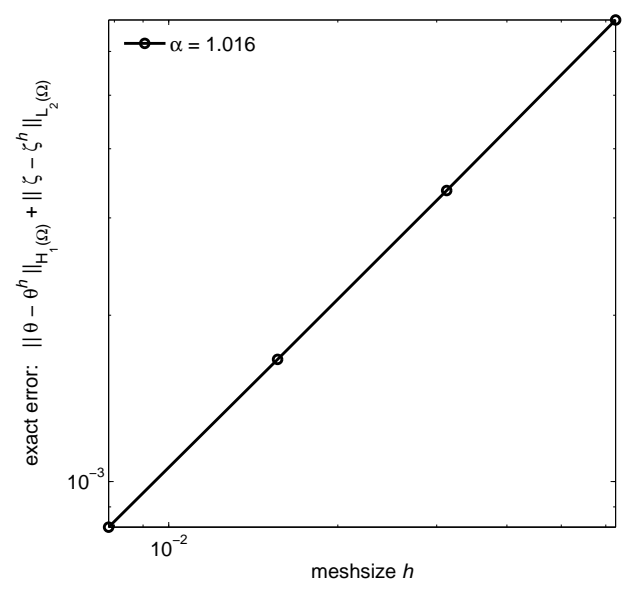

(b) A priori error as a function of meshsize for plate thickness $t=10^{-1}$.

Fig. 8 . 


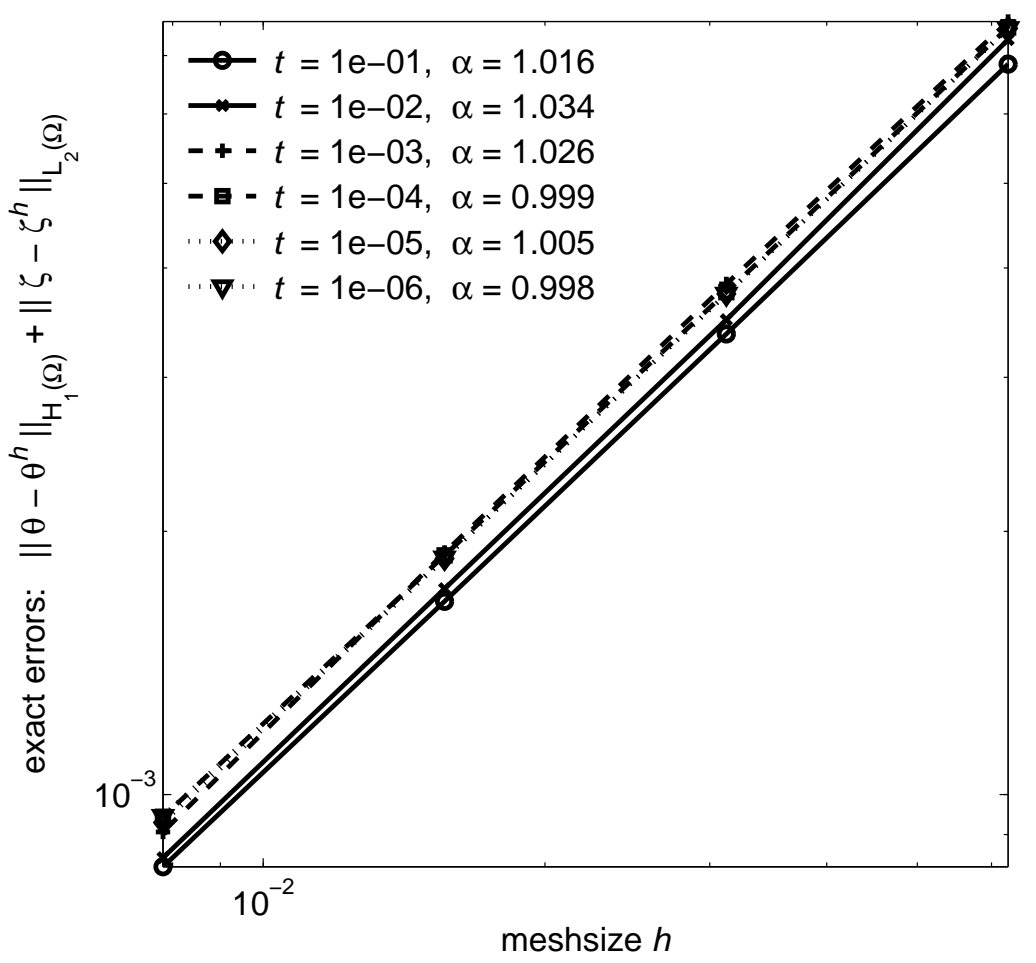

Fig. 9. A priori errors, for a set of different plate thicknesses, as a function of meshsize.

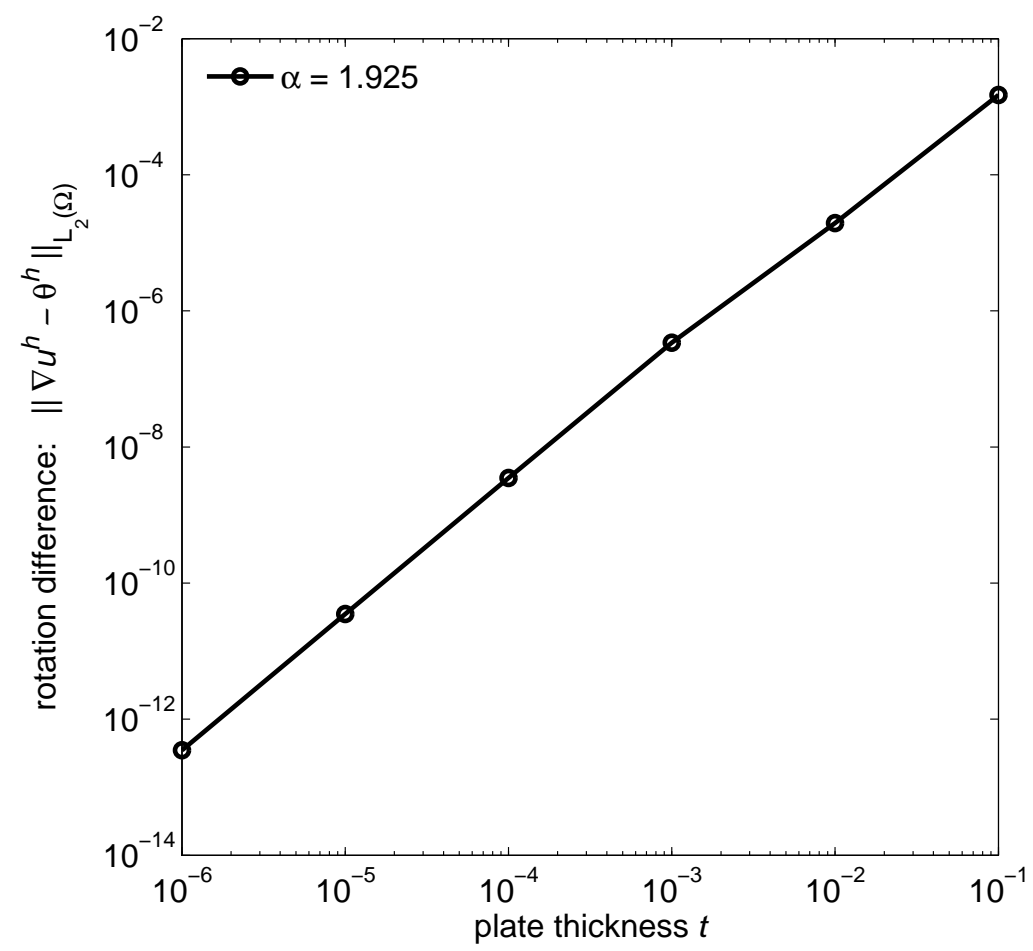

Fig. 10. Difference between the displacement gradient and the rotation vector as a function of plate thickness. 


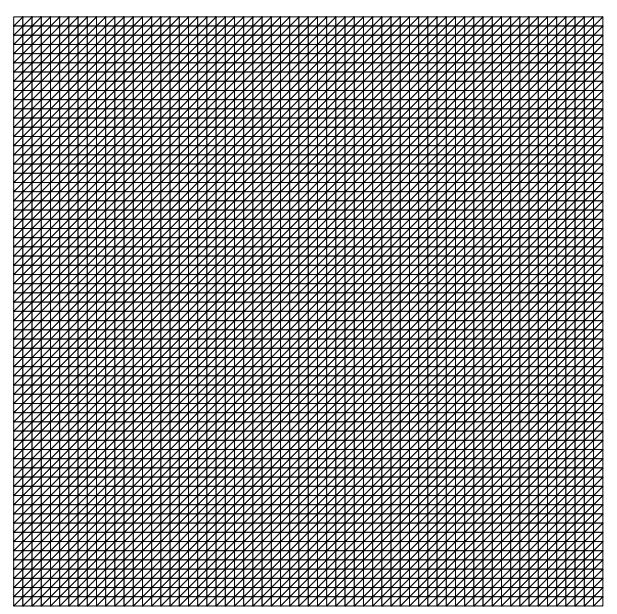

(a) 8 192-element mesh with directionality.

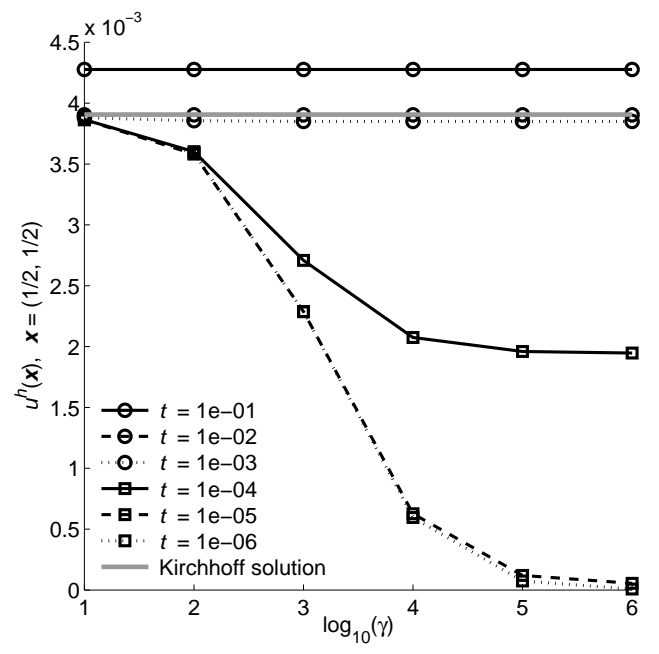

(b) Midpoint displacements for a set of plate thicknesses as a function of the stability parameter.

Fig. 11.

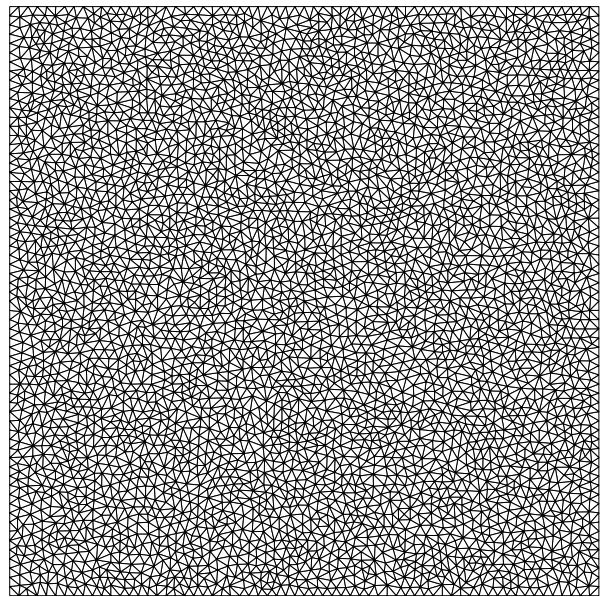

(a) 8 198-element unstructured mesh.

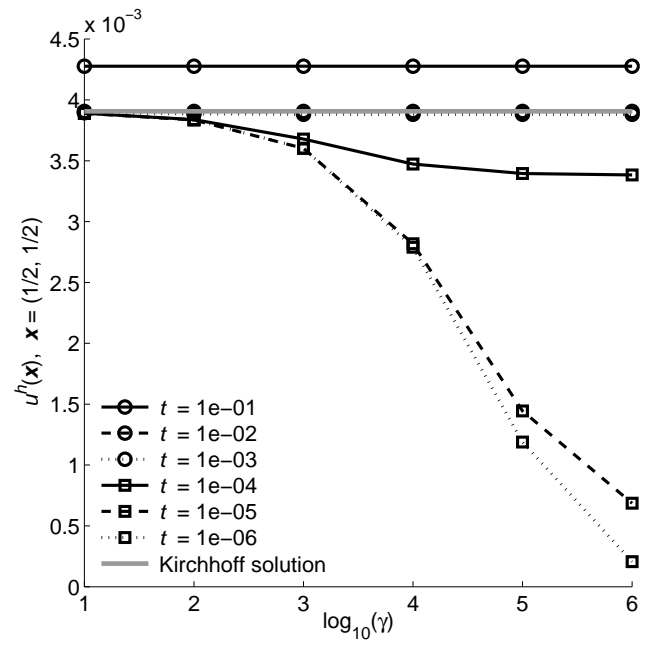

(b) Midpoint displacements for a set of plate thicknesses as a function of the stability parameter.

Fig. 12. 


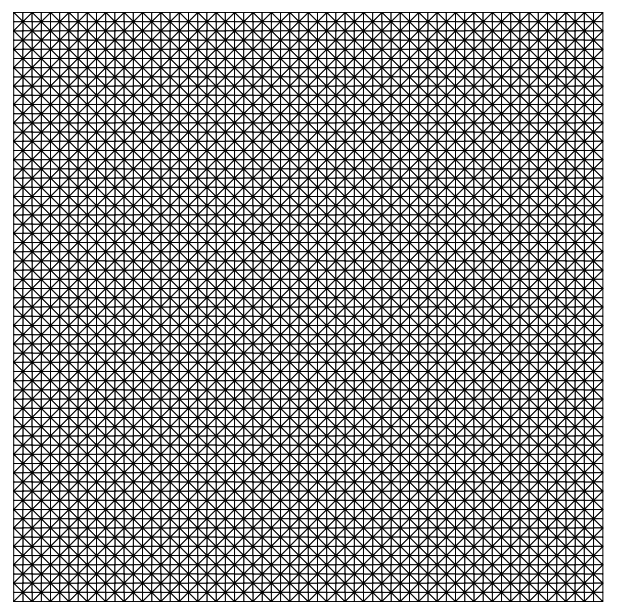

(a) 8 192-element criss-cross mesh.

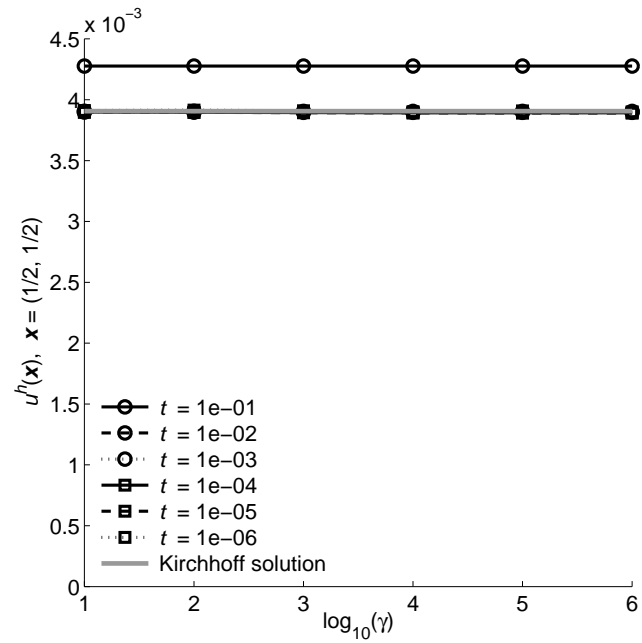

(b) Midpoint displacements for a set of plate thicknesses as a function of the stability parameter.

Fig. 13. 